\title{
Seroprevalence of Bordetella pertussis antibodies in mothers and their newborn infants
}

\author{
BERNARD GONIK ${ }^{1}$, KAROLINE S. PUDER ${ }^{1}$, NATHAN GONIK ${ }^{2}$, \& MICHAEL KRUGER ${ }^{1}$ \\ ${ }^{1}$ Department of Obstetrics and Gynecology, Wayne State University School of Medicine, Detroit, MI, USA, and ${ }^{2}$ School of \\ Public Health, University of Michigan, Ann Arbor, MI, USA
}

(Received 15 fuly 2004; accepted 1 October 2004)

\begin{abstract}
Background. Pertussis is a highly communicable, vaccine-preventable respiratory disease. Although the largest number of reported cases is among young infants, the most rapidly increasing incidence in the USA is in adolescents and young adults. Importantly, adult family members are the likely major reservoir, infecting susceptible infants before completion of childhood vaccination. We studied maternal-neonatal paired blood samples for the presence of pertussis-related antibodies to assess level of immunity and passive transplacental antibody passage.

Methods. Unselected maternal-neonatal cord blood samples were collected from 101 term deliveries in a single urban uninsured/underinsured hospital setting. Sera were analyzed for anti-pertussis toxin (PT), filamentous hemagglutinin (FHA) and pertactin (PRN) IgG antibodies by enzyme-linked immunosorbent assay (ELISA). Antibody titers were calculated using reference line methodology. Antibody values were log-transformed to establish geometric mean titers (GMT) for analysis. Student's $t$-test, Mann-Whitney, Pearson correlation and chi square were used for statistical comparisons as appropriate. Results. Mean (SD) maternal age, gestational age and birth weight were 26.8 (6.8) years, 38.9 (1.4) weeks and 3239 (501) g, respectively. Detectable maternal levels of anti-PT, FHA and PRN were found in $34.7 \%, 95.0 \%$ and $80.2 \%$, respectively. Maternal GMT (SD) for PT, FHA and PRN were 4.4 (2.6), 26.6 (3.1) and 12.3 (2.9), respectively. There was no significant relationship between PT, FHA or PRN detection or antibody GMT and maternal age. Maternal anti-PT, FHA and PRN were highly correlated with neonatal cord blood values.

Conclusion. Despite previous childhood immunization, a large number of parous women have low or undetectable pertussis-related antibody levels, suggesting susceptibility to infection. Even with efficient transplacental passage of these antibodies, neonates similarly have limited measurable protection as detected by cord blood sampling. These data support the need for adolescent or adult vaccination against Bordetella pertussis. Healthcare providers and their clients should be aware of the risk for infant infection via family member transmission.
\end{abstract}

Keywords: Pertussis, immunity, pregnancy, neonatal

\section{Introduction}

Pertussis is a highly communicable, vaccine-preventable respiratory disease. Although the largest number of reported cases is among young infants, the most rapidly increasing incidence in the USA is in adolescents and young adults $[1,2]$. Importantly, adult family members are the likely major reservoir, infecting susceptible infants before completion of childhood vaccination. We studied maternal-cord blood paired samples for the presence of pertussisrelated antibodies to assess level of immunity and passive transplacental antibody passage.

\section{Methods}

Unselected maternal-neonatal cord blood samples were collected from 101 term deliveries in a single urban uninsured/underinsured hospital setting. Although detailed demographic data were not available, the patient population was more than $80 \%$ African-American of USA origin. Samples were stored at $-20^{\circ} \mathrm{C}$ until analyzed for antipertussis toxin (PT), filamentous hemagglutinin (FHA) and pertactin (PRN) IgG antibodies by enzyme-linked immunosorbent assay (ELISA), as follows.

Correspondence: Bernard Gonik, Department of Obstetrics and Gynecology, Wayne State University School of Medicine, 6071 W. Outer Drive, Detroit, Michigan 48235, USA. Tel: 313-966-3246. Fax: 313-966-4296. E-mail: bgonik@dmc.org

ISSN 1064-7449 print/ISSN 1098-0997 online (C) 2005 Taylor \& Francis Group Ltd DOI: $10.1080 / 10647440500068289$ 
The antigen of interest was adsorbed onto a polystyrene microtiter plate. Dilutions of human sera were added to the PT-, FHA- or PRN-coated plates and the antibodies specific for each bound to the coated antigen. Sera were diluted with phosphate-buffered saline containing $0.2 \%$ bovine serum albumin (BSA), and seven serial twofold dilutions were performed. Each plate included the standard and two positive controls. Standard and controls were pools of human sera. The standards used were internal reference sera calibrated against FDA control serum lots, defined as containing $200 \mathrm{EU} /$ $\mathrm{ml}$ of IgG antibody (for PT and FHA) and $90 \mathrm{EU} / \mathrm{ml}$ (for PRN). The bound antibodies were detected using a goat anti-human IgG alkaline phosphatase labeled antibody followed by p-nitrophenyl phosphate substrate. The color developed was directly proportional to the amount of antibody present in the serum. Absorbance readings were measured using a spectrophotometer capable of reading microtiter plates. The IgG antibody titers were calculated relative to the reference, using the reference line method. Antibody levels were expressed in $\mathrm{EU} / \mathrm{ml}$. The assay cut-offs were set at $5 \mathrm{EU} / \mathrm{ml}$. The between-day coefficient of variation was $<25 \%$ for each assay. Antibody values were subsequently logtransformed to establish geometric mean titers (GMT) for further analyses.

Data were analyzed using SPSS 11.0 for Windows (SPSS, Chicago, IL, USA). Student's $t$-test, MannWhitney U test, Pearson correlations and Pearson chi square were used for statistical comparisons as deemed appropriate.

\section{Results}

Mean (SD) maternal age, gestational age and birth weight were 26.8 (6.8) years, 38.9 (1.4) weeks and 3239 (501) g, respectively. Detectable maternal levels of anti-PT, FHA and PRN were found in $34.7 \%, 95.0 \%$ and $80.2 \%$ of samples, respectively. Detectable cord blood levels of anti-PT, FHA and PRN were seen in $44.6 \%, 93.2 \%$, and $80.6 \%$ of samples, respectively. Maternal and cord blood geometric mean titers for anti-PT, FHA and PRN are shown in Table I. There was no significant relationship between anti-PT, FHA or PRN detection or geometric mean titer and maternal age.

Table I. Geometric mean (SD) values for pertussis antibodies.

\begin{tabular}{lcc}
\hline Antibody & Maternal $(n=101)$ & Cord blood $(n=103)$ \\
\hline PT & $4.4(2.6)$ & $5.6(3.0)$ \\
PRN & $12.3(2.9)$ & $10.2(3.2)$ \\
FHA & $26.6(3.1)$ & $32.0(3.2)$ \\
\hline
\end{tabular}

Maternal anti-PT, FHA, and PRN were significantly correlated $(p<0.001)$ with neonatal cord blood values; $r=0.98,0.90$ and 0.96 , respectively.

\section{Discussion}

Pertussis is a highly communicable, vaccine-preventable respiratory disease caused by the organism Bordetella pertussis. In the USA, the incidence of reported cases has dramatically increased over the last 10 years [1]. The highest incidence of new pertussis cases is among infants (Table II). However, the most rapidly increasing incidence has been noted among adolescents and young adults $[1,2]$. The epidemiology of pertussis in this latter population is not well defined, and clinicians commonly overlook this diagnosis in otherwise healthy patients presenting with chronic cough [3]. A key concern is that adult hosts act as a reservoir for the organism, allowing for infection of pre-vaccinated, susceptible newborns and young children, in whom morbidity and mortality are more pronounced [4].

Despite the general perception that either natural or vaccine-induced pertussis immunity is long lasting and therefore protective, recent studies have demonstrated adult susceptibility even in highly vaccinated communities [5]. Serological surveillance data likewise highlight waning immunity as adolescence approaches [1]. The data presented in our study, specifically examining seroprevalence of Bordetella pertussis antibodies in an obstetric and newborn population, add support to these concerns regarding adult susceptibility, and are in general agreement with recently reported observations [6,7]. Even with efficient transplacental passage of passive immunity to the newborn, our results also underscore the possible lack of protection available to the newborn immediately after delivery.

The antibodies studied in this investigation are commonly examined in relation to Bordetella pertussis infection and vaccine efficacy $[7,8]$. However, a clear understanding of the exact mechanisms underlying immunity to pertussis is lacking. It is likely that immunity to pertussis involves multiple humoral and cellular responses that are not directed against a single protective antigen. Anti-PT has been corre-

Table II. Average annual incidence rates of pertussis in the USA, 1997-2000 [1].

\begin{tabular}{lc}
\hline Age (years) & Cases $($ per 100,000) \\
\hline$<1$ & 55.5 \\
$1-4$ & 5.5 \\
$5-9$ & 3.6 \\
$10-19$ & 5.5 \\
$\geqslant 20$ & 0.8 \\
\hline
\end{tabular}


lated with clinical protection against Bordetella pertussis infection in humans, but an absolute cutoff value for protection has not been determined [5]. Similarly, the presence of anti-PRN correlates crudely with clinical immunity against disease [5]. These two antibodies seem to be specific for Bordetella pertussis, with no cross-reactivity to other species [5]. Conversely, anti-FHA has been shown to cross-react with Bordetella parapertussis, and therefore the significance of this antibody remains to be fully elucidated [5].

The parturients and neonates in our study with antibodies below detectable levels probably represented susceptible individuals. For anti-PT, this comprised almost two thirds of the maternal samples and almost one half of the newborn cord blood specimens. Applying one reported set of criteria for anti-PT and PRN cut-off levels for serologic evidence of protection, the overwhelming majority of our serologically positive subjects would also be deemed moderately to highly susceptible to infection [9].

Despite the controversy regarding absolute antibody values and host protection, our data, in combination with recent epidemiologic studies reporting significant rises in clinical pertussis cases, should stimulate additional research in this area $[1,2,10]$. Many authorities have already argued that routine adolescent and adult re-vaccination with an acellular pertussis agent should be considered, to decrease disease burden and to control transmission to susceptible newborn infants $[11,12]$. At a minimum, healthcare providers and their clients should be aware of the risk for infant infection via family member transmission. This should lead to more careful evaluation and testing of household contacts with atypical coughs, with more attention to the use of efficacious antimicrobial therapies and prophylaxis where indicated.

\section{Acknowledgment}

We would like to acknowledge our gratitude to Dominique Descamps, Emmanuel Dael and the Glaxo SmithKline Biologicals Laboratory in Rixensart, Belgium, for their assistance in running the antibody assays.

\section{References}

1. Centers for Disease Control and Prevention. Pertussis - USA, 1997-2000. MMWR recomm Rep 2002;51(4):73-76.

2. Yih WK, Lett SM, des Vignes FN, et al. The increasing incidence of pertussis in Massachusetts adolescents and adults, 1989-1998. J Infect Dis 2000;182:1409-1416.

3. Wright SW. Pertussis infection in adults. South Med J 1998;91:702-708.

4. Centers for Disease Control and Prevention. Guidelines for the control of pertussis outbreaks. Atlanta, GA, USA: Centers for Disease Control and Prevention; 2000.

5. Van de Wielen $M$, Van Damme P, Van Herck K, et al. Seroprevalence of Bordetella pertussis antibodies in Flanders (Belgium). Vaccine 2003;21:2412-2417.

6. Healy CM, Munoz FM, Rench MA, et al. Prevalence of pertussis antibodies in maternal delivery, cord, and infant serum. J Infect Dis 2004;190:335-340.

7. Belloni C, DeSilvestri A, Tinelli C, et al. Immunogenicity of a three-component acellular pertussis vaccine administered at birth. Pediatrics 2003;111:1042-1045.

8. Storsaeter J, Hallender HO, Gustafsson L, Olin P. Levels of anti-pertussin antibodies related to protection after household exposure to Bordetella pertussis. Vaccine 1998;16:1907-1916.

9. Cherry JD, Gornbein J, Heininger U, Stehr K. A search for serologic correlates of immunity to Bordetella pertussis cough illnesses. Vaccine 1998;16:1901-1906.

10. Guris D, Strebel PM, Bardenheier B, et al. Changing epidemiology of pertussis in the USA: increasing reported incidence among adolescents and adults, 1990-1996. Clin Infect Dis 1999;28:1230-1237.

11. Edwards KM. Pertussis: an important target for maternal immunization. Vaccine 2003;21:3483-3486.

12. Health Canada. National consensus conference on pertussis. Canada Communicable Disease Report 2001;2953:1-33. 


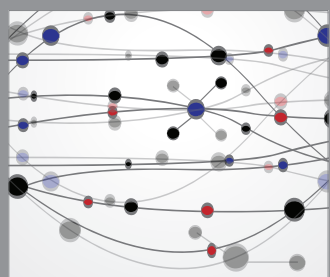

The Scientific World Journal
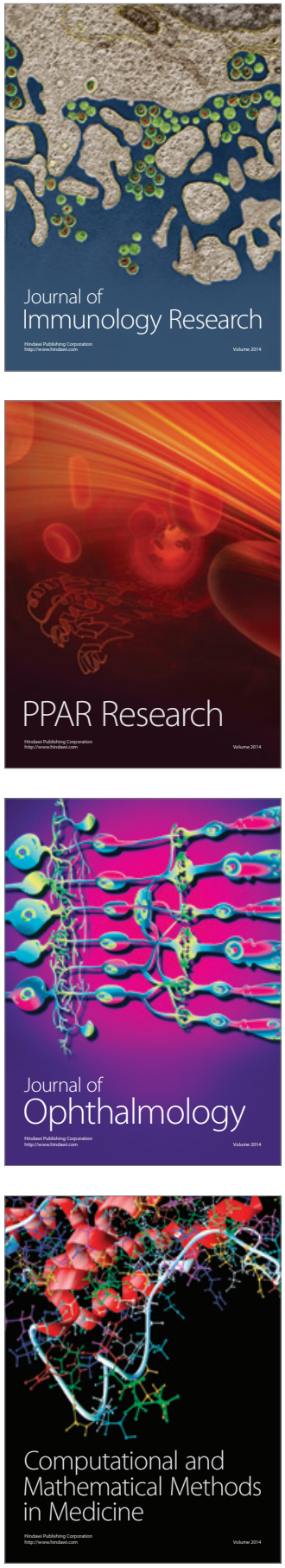

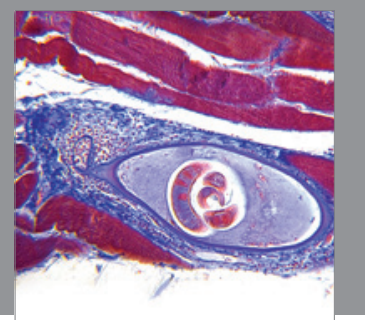

Gastroenterology

Research and Practice
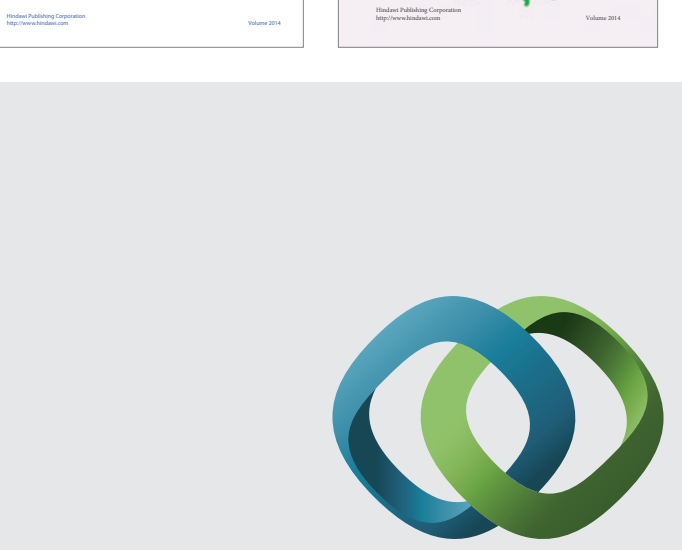

\section{Hindawi}

Submit your manuscripts at

http://www.hindawi.com
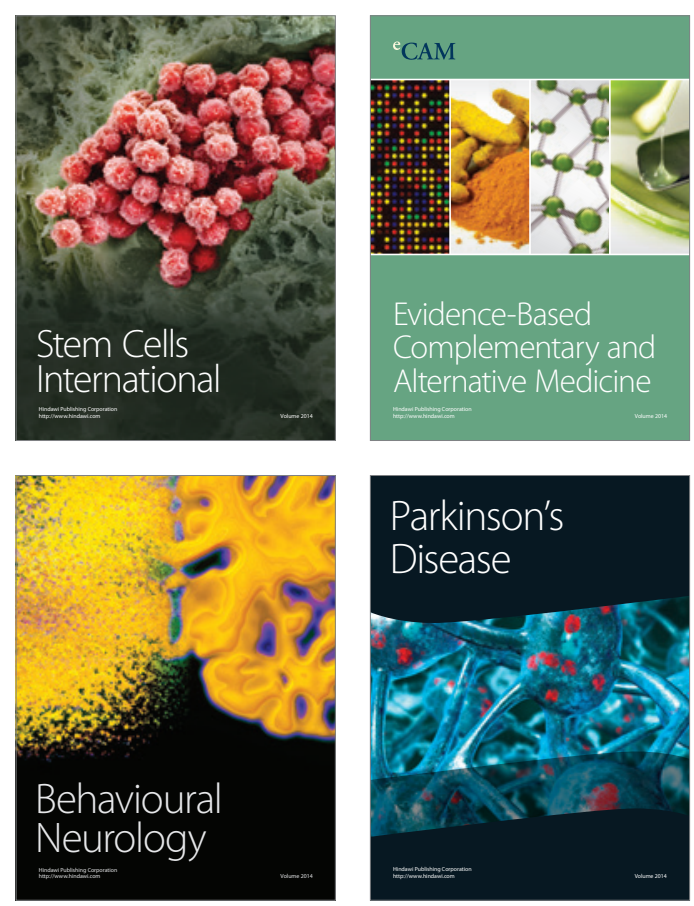

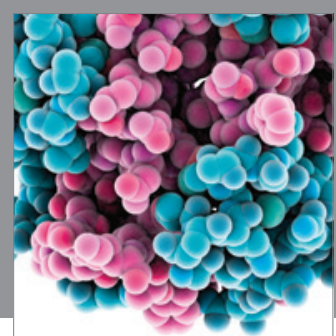

Journal of
Diabetes Research

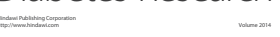

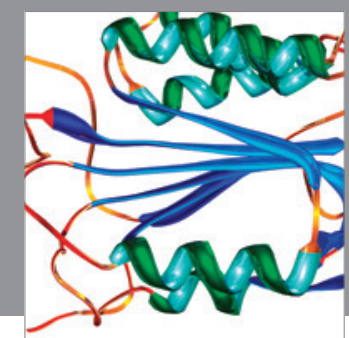

Disease Markers
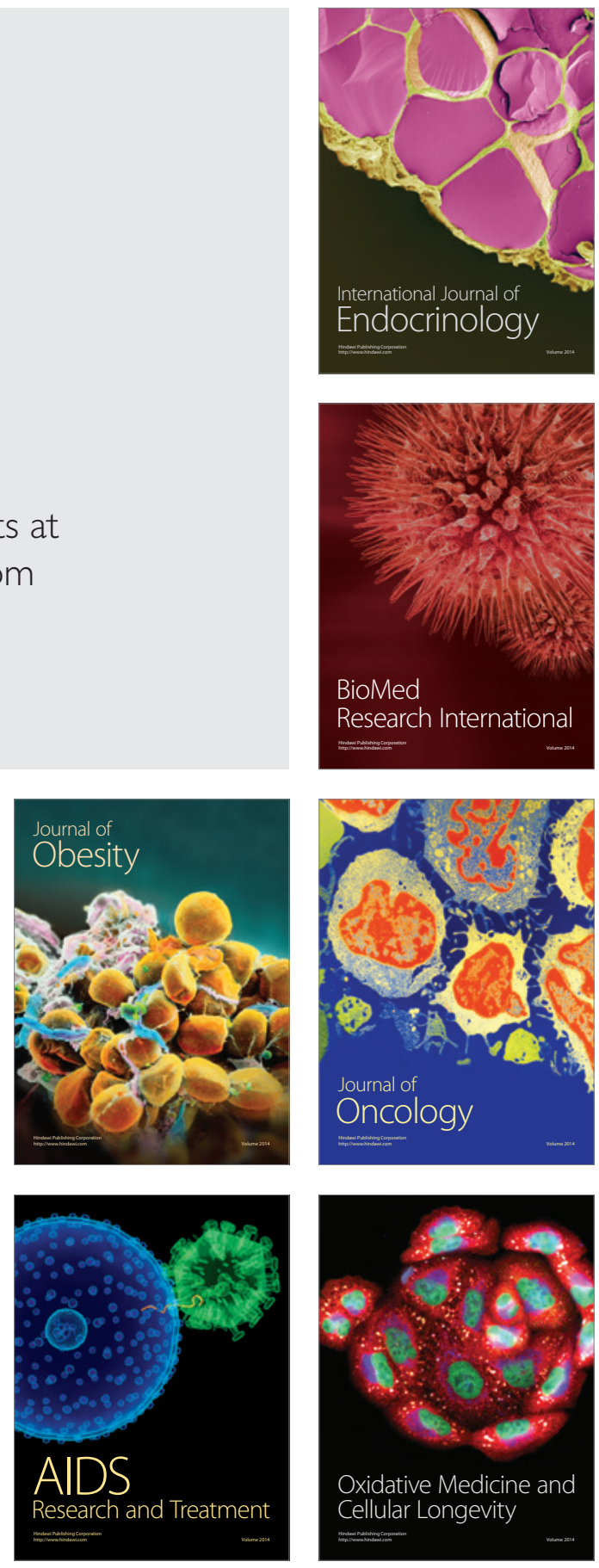